\title{
Community niche predicts the functioning of denitrifying bacterial assemblages
}

\author{
Salles, J F ; Poly, F ; Schmid, B ; Le Roux, X
}

\begin{abstract}
Predicting biodiversity effects on ecosystem functioning requires adequate evaluation of the mechanisms explaining why more diverse systems could perform better than less diverse ones. In this context, tackling functional diversity has become an important issue. Even though the aggregation of species into functional groups supposes niche differences among groups, the concept of niche has not been fully exploited in the context of the biodiversity-ecosystem functioning research. Here we report the results of microcosm experiments where we used bacteria as a model to explore whether niche differences among species provide a good estimation of community functioning. For that we used experimental communities of denitrifying bacterial species and investigated the effects of bacterial diversity on two community processes, denitrification and anaerobic CO2-production. We first measured the activities of 16 bacterial species grown individually on six different carbon sources. We then used the same set of species to assemble communities varying in both species richness and composition in microcosms containing a mixture of all six carbon sources. The performances of individual species on individual carbon sources were used to calculate, for each process measured, an a priori index called "community niche" that accounted for the performances of the species present in a given community across the entire range of the six resources. We found that species richness had a positive but small effect on both community processes whereas community niche explained a much larger proportion of the variation. According to the results of a path analysis, community niche was the main driver for the corresponding community process, but species richness affected community niche and thus had an indirect effect on denitrification and $\mathrm{CO} 2$ production. In addition to community niche, the presence of particular bacterial species also influenced community functioning, indicating that other effects than the capacity to use carbon sources played a, albeit minor, role in our experiment. Our study provides evidence for the importance of resource niches in shaping biodiversity-ecosystem functioning relationships of bacterial communities.
\end{abstract}

DOI: https://doi.org/10.1890/09-0188.1

Posted at the Zurich Open Repository and Archive, University of Zurich

ZORA URL: https://doi.org/10.5167/uzh-26837

Journal Article

Originally published at:

Salles, J F; Poly, F; Schmid, B; Le Roux, X (2009). Community niche predicts the functioning of denitrifying bacterial assemblages. Ecology, 90(12):3324-3332.

DOI: https://doi.org/10.1890/09-0188.1 
1 COMMUNITY NICHE PREDICTS THE FUNCTIONING OF DENITRIFYING

2 BACTERIAL ASSEMBLAGES

3

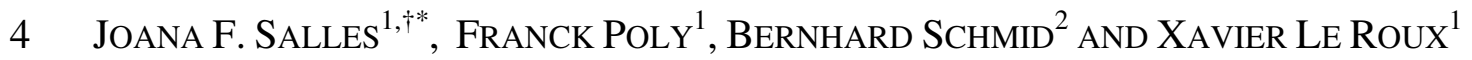

5

$6{ }^{1}$ Université de Lyon, INRA, CNRS, Université Lyon 1, Ecologie Microbienne (UMR 5557, USC

7 1193), bat. G. Mendel, 43 boulevard du 11 novembre 1918, 69622 Villeurbanne, France

$8{ }^{2}$ Institute of Environmental Sciences, University of Zurich, Winterthurerstrasse 190, 8057 Zurich,

$9 \quad$ Switzerland

10

11 Running title: community niche drives bacterial functioning

12 Submitted to: Ecology

13 Manuscript type: Article

14 Abstract: 325 words; 29 references; 5 figures; 1 table; 2 appendices

16 * To whom correspondence should be addressed:

17 Joana Falcão Salles

$18 \dagger$ Present address: Microbial Ecology, University of Groningen, Kerklaan 30, Haren, the

19 Netherlands, $9751 N N$

20 Tel: (31) 50363 2162; Fax: (31) 503632154

21 Email: j.falcao.salles@rug.nl 


\section{$1 \quad$ AbSTRACT}

2 Predicting biodiversity effects on ecosystem functioning requires to adequately evaluate the

3 mechanisms explaining why more diverse systems could perform better than less diverse ones. In

4 this context, tackling functional diversity has become an important issue. Even though the

5 aggregation of species into functional groups supposes niche differences among groups, the

6 concept of niche has not been fully exploited in the context of the biodiversity-ecosystem

7 functioning research. Here we report the results of microcosm experiments where we used

8 bacteria as a model to explore whether niche differences among species provide a good

9 estimation of community functioning. For that we used experimental communities of denitrifying

10 bacterial species and investigated the effects of bacterial diversity on two community processes,

11 denitrification and anaerobic $\mathrm{CO}_{2}$-production. We first measured the activities of 16 bacterial

12 species grown individually on six different carbon sources. We then used the same set of species

13 to assemble communities varying in both species richness and composition in microcosms

14 containing a mixture of all six carbon sources. The performances of individual species on

15 individual carbon sources were used to calculate, for each process measured, an a-priori index

16 called "community niche" which accounted for the performances of the species present in a given

17 community across the entire range of the six resources. We found that species richness had a

18 positive but small effect on both community processes whereas community niche explained a

19 much larger proportion of the variation. According to the results of a path analysis, community

20 niche was the main driver for the corresponding community process but species richness affected

21 community niche and thus had an indirect effect on denitrification and $\mathrm{CO}_{2}$ production. In

22 addition to community niche, the presence of particular bacterial species also influenced

23 community functioning, indicating that other effects than the capacity to use carbon sources

24 played a, albeit minor, role in our experiment. Our study provides evidence for the importance of 
1 resource niches in shaping biodiversity-ecosystem functioning relationships of bacterial

2 communities.

3

4 Key words: biodiversity-ecosystem function relationships, community niche, carbon resource-

5 use, bacterial diversity, denitrification, anaerobic $\mathrm{CO}_{2}$ production

6

7

8

9

10

11

12

13

14

15

16

17

18

19

20

21

22

23

24 

studies mainly focusing on trophic interactions in guilds or on factors limiting the species 22 richness in biological communities, it has never been fully exploited in the context of the BEF 23 within trophic levels (Leibold 1995).

\section{INTRODUCTION}

Identifying the mechanisms explaining why more diverse systems could perform better than less diverse ones has become an essential issue in studies focusing on biodiversityecosystem functioning (BEF) relationship. In this context, tackling functional diversity among species has been of great importance (Lavorel and Garnier 2002, Petchey and Gaston 2006). When aggregating similar species into functional groups, we assume that functional differences among these groups would affect the functioning of communities. Considering niche as the impact species have on resource use (according to Elton's and later MacArthur \& Levin's definition; Leibold 1995), the breadth of resources used by each species (species niche breadth) and more particularly, the level of complementarity observed between species niches in an assembled community, is expected to have an impact on how species diversity affects ecosystem processes. Indeed, resource partitioning is considered as one of the main mechanisms describing species coexistence (resource-ratio theory, Tilman 1982) and has been regarded as a major mechanism explaining the positive effects of diversity in BEF experiments (Loreau and Hector 2001). However, the latter is based on indirect evidence supported by the additive partitioning, when the yields of communities with multiple species are on average higher than expected on the basis of the yield of their monocultures (Loreau and Hector 2001). In order to provide direct and mechanistic evidence of niche differentiation, a possible approach would be to define the niche breadths for each species present in an assemblage, and use these data to characterize the niche of that community. Whereas the concept of community niche has been used in previous ecological 
Here we report the results of microcosm experiments where we used bacteria species as a

2 model to explore whether niche differences among species provide a good estimation of

3 community functioning. The value of bacterial model systems for testing ecological theories has

4 been recently underlined (Jessup et al. 2004, Prosser et al. 2007), in particular for unravelling the

5 mechanisms determining BEF relationships. Bacteria are key players in nitrogen, carbon,

6 phosphorus and sulphur cycles; and resource-driven interactions among bacterial species are

7 likely to have a great impact on community and ecosystem functioning (Naeem et al. 2000). In

8 addition, for technical and biological reasons, characterizing niche breadth for a range of species

9 can be easier for bacteria than for higher organisms.

10 For our experiments, we focused on bacterial species involved in denitrification, an

11 important process in nitrogen cycling which is carried out by microorganisms that differ in their

12 affinities for and rates of processing different substrates (Cavigelli and Robertson 2001, Philippot

13 and Hallin 2005). We opted for the use of microcosm experiments with bacterial communities

14 because of the simplicity of these systems which allows a full description of species performance

15 across a range of well-defined single-resource environments and consequently the

16 characterization of species niche breadths. These species niche breadths may allow defining the

17 niche breadth of communities and furthermore, predicting their performance in mixed-resource

18 environments. In this context, our objectives were to assess (1) whether niche breadths of species

19 could be used to compute an index that represents the niche breadth of a community, and (2)

20 whether this index would provide a better understanding of the role of species diversity on the

21 functioning of a community. Our hypothesis was that a measure of community niche should

22 represent the overall potential of the community to extract resources from the environment and

23 should therefore be more directly related to ecosystem functioning than are measures of species

24 diversity. 
In order to test our hypothesis, we run two experiments. The first one consisted in

2 determining the performance of a set of denitrifying species, growing alone on individual carbon

3 sources. Because the bacterial species used are commonly found in terrestrial ecosystems, we

4 selected carbon sources which also occur abundantly in soil, either due to organic matter

5 degradation or plant exudation. The results from this experiment provided us the niche breadths

6 for each species studied. In the second experiment we evaluated the effect of species diversity on

7 community functioning by manipulating bacterial communities at different levels of species

8 richness, and growing them on a mixture of the carbon sources used in the first experiment. We

9 calculated the community niche for each bacterial assemblage by taking the sum over all carbon

10 sources of the maximal performance observed for a species in the assemblage on each individual

11 carbon source. Thus, consider an assemblage of two species $\mathrm{X}$ and $\mathrm{Y}$, growing in a mixture of

12 two carbon sources A and B. If when growing on individual substrates, species $\mathrm{X}$ has the highest

13 performance on the first source, and species $\mathrm{Y}$ the highest on the second source, then the

14 community niche of this assemblage (on this resource mixture) would be the sum of the

15 performance of species $\mathrm{X}$ on source $\mathrm{A}$ and the performance of species $\mathrm{Y}$ in source $\mathrm{B}$. Once

16 community niche was calculated for each one of the bacterial assemblages, it was used in

17 statistical models, together with species richness and initial species composition, to determine

18 their importance in explaining the overall community performances observed in the second

19 microcosm experiment.

20 As our results will show, species richness had a positive but small effect on community

21 processes whereas community niche explained a much larger proportion of the variation. Thus,

22 by aggregating resource-use characteristics of species in a community into a synthetic descriptor

23 of community niche, we were able to better predict the observed variations in community

24 functioning. In addition to community niche, the presence of particular bacterial species also 
1 influenced community functioning, indicating that other effects than the capacity to use the

2 carbon sources provided in culture medium played a role in our experiment. This study

3 demonstrates the importance of complementarity for resource use among species in explaining

4 the enhanced performance of diverse bacterial communities.

\section{MeTHODS}

7 Bacterial species

8 The 16 bacterial species used in our microcosms were chosen according to: (i) their ability

9 to denitrify; (ii) their common occurrence in the soil or rhizosphere; and (iii) their wide

10 distribution over different bacterial phyla. The bacterial species were identified by sequencing

11 their respective 16S rRNA gene, which is broadly used for taxonomical purposes, and comparing

12 it against a public database (NCBI, http://www.ncbi.nlm.nih.gov). Thus, the bacterial species

13 belonged to the following taxa: Achromobacter xylosoxidans subsp. denitrificans D35;

14 Azospirillum lipoferum A5; Bacillus cereus A19; Bacillus weihenstephanensis A20; Burkholderia

15 cepacia G7; Citrobacter braakii A7; Ensifer adhaerens A1; Klebsiela pneumoniae A18;

16 Ochrobactrum sp. A6; Ochrobactrum sp. A17; Ochrobactrum sp. A22; Paracoccus denitrificans

17 G11; Pseudomonas aeruginosa G16; Pseudomonas fluorescens A14; Pseudomonas stutzeri A16;

18 and Pseudomonas stutzeri A24. Species assigned to the same taxon were further characterized by

19 BOX-PCR, according to the methodology described by Bathe et al. (2006) (Appendix A). The

20 BOX is a repetitive DNA element which is present at strain-specific intergenic positions

21 throughout the genomes and therefore can be used to generate genomic fingerprints (barcodes)

22 that allow the classification of bacterial isolates at species, subspecies and strain level. By using

23 this method we were able to determine that bacterial isolates assigned to the same species by

24 sequencing the 16S rRNA gene were indeed different strains. 
The experimental microcosms consisted of 150-ml plasma flasks sealed with rubber

4 stoppers and containing $50 \mathrm{ml}$ of minimal medium M9 (Sambrook et al. 1989) supplemented with

$5 \quad 0.2 \mathrm{M} \mathrm{KNO}_{3}$ and selected carbon sources (total $\mathrm{C}$ concentration of $1.6 \mathrm{mg} \mathrm{C} \mathrm{l}^{-1}$ ). We replaced the

6 atmosphere of the flasks by a 90:10 mixture of $\mathrm{He}-\mathrm{C}_{2} \mathrm{H}_{2}$ to provide anaerobic conditions for

7 denitrification and to inhibit of $\mathrm{N}_{2} \mathrm{O}$-reductase activity, allowing us to quantify the amount of

$8 \quad \mathrm{~N}_{2} \mathrm{O}$ and $\mathrm{CO}_{2}$ produced by the bacterial species or species assemblages. Flasks were incubated at

$928^{\circ} \mathrm{C}$ and $160 \mathrm{rpm}$. In the microcosms used to define the species niche breadth, each bacterial

10 species was grown on flasks containing individual carbon sources. Six sources were used, D-

11 cellobiose, maltose, L-fucose, L-malic acid, L-glutamine and fumaric acid. They were chosen

12 because of their occurrence in soil, where the selected bacterial were originally obtained from.

13 The bacterial inoculum consisted of cells at the exponential growth phase, growing anaerobically

14 on the same medium containing all six carbon sources. Before inoculation, cells were harvested

15 by centrifugation, washed with sterile PBS buffer and let to starvation for $2 \mathrm{~h}$ at room

16 temperature on PBS. The starvation step was necessary to allow cells to consume their resources

17 completely prior to inoculation on individual sources, and avoid biases when characterizing

18 species performances. Species were inoculated in the single C-source microcosms to obtain a

19 final cell density of $\mathrm{OD}_{560}=0.002$. This experiment was carried out twice.

20 The experiment used to evaluate the effect of bacterial diversity on community functioning

21 was performed in a similar manner except for two points. The first point relates to the carbon-

22 resource environment, which in this case corresponded of a mixture with equal C-concentration

23 (final concentration: $1.6 \mathrm{mg} \mathrm{C}^{-1}$ ) of the six carbon sources used for the previous experiments.

24 The second point concerns the inoculum density, which was kept at $\mathrm{OD}_{560}=0.002$ for the total 
1 community in a microcosm as previously, leading to a decrease in per-species inoculum with

2 increasing species richness. Thus, each microcosm was inoculated with denitrifying bacterial

3 species at a cell density of $\mathrm{OD}_{560}=0.002 / s$, where $s$ is the number of species in the community

4 inoculum. Therefore, at the beginning of the microcosm experiments, all bacterial assemblages

5 contained approximately the same total cell density, regardless of their species richness and

6 composition.

Measurements

9 The performances of individual species and bacterial communities in the respective

10 microcosm experiments were determined by measuring both $\mathrm{N}_{2} \mathrm{O}$ and $\mathrm{CO}_{2}$ production (in $\mu \mathrm{g}-\mathrm{N}$

11 or $\mu \mathrm{g}-\mathrm{C} \mathrm{ml}^{-1} \mathrm{~h}^{-1}$ ), which will be referred to throughout the text as denitrification and (anaerobic)

$12 \mathrm{CO}_{2}$-production, respectively. Both measurements were performed, after collecting gas samples

13 on regular basis up to 8 days after inoculation, using a gas chromatograph (Agilent P200, USA).

14 In addition, at each sampling point, we collected $1 \mathrm{ml}$ of culture, which we used to determine the

15 total number of bacterial cells with a flow cytometer (BD FACSCalibur, USA), providing us with

16 information about possible effects of species diversity or composition on the cell density in each

17 microcosm.

19 Experimental design

20 We assembled bacterial communities following a "broken stick" design, which allows

21 separation of the effects of bacterial richness and composition (Bell et al. 2005), because each

22 species combination is nested in the combinations of higher species richness. This design consists

23 in defining a list or "stick" where all 16 species are put in random order without replication. The 
1 complete stick corresponds to the assemblage with all species. We then divided this stick in the

2 middle, generating two sticks of 8 species that corresponded to two assemblages, one containing

3 eight species present in one half of the stick and the other assemblage containing the remaining

4 eight species. Each 8-species stick was further divided in the middle; creating four 4-species

5 sticks and so on, until the 16 monocultures were reached. In order to distinguish the effects of

6 species richness from composition, it is essential that for a given level of species richness,

7 assemblages with different bacterial composition are created. We therefore made three 16-species

8 sticks, each one containing the same species but in different order. These sticks were "broken",

9 providing 6 assemblages with 8 species, 12 with 4 species, 24 with 2 species and 16

10 monocultures. In order to increase the number of communities with 8 species, we added 3 extra

11 assemblages, each consisting of the first four and the last four species of each one of the 16-

12 species sticks. The 16 monocultures were replicated twice and the single 16-species assemblage

13 three times, giving a total of 80 microcosms.

\section{Community niche}

16 The niche of a given community (community niche, $\mathrm{CN}$ ) was calculated based on the

17 performance of each species on each one of the carbon sources, according to the formula:

$$
C N=\sum_{i=1}^{6} \max _{j=1}^{n}\left(P_{i j}\right)
$$

19 where $\mathrm{P}_{i j}$ is the performance (i.e. denitrification or anaerobic $\mathrm{CO}_{2}$-production activity, in $\mathrm{mgN}$ -

$20 \mathrm{~N}_{2} \mathrm{O}$ or $\mathrm{mgC}-\mathrm{CO}_{2} \mathrm{ml}^{-1} \mathrm{~h}^{-1}$ ) of species $j$ on carbon source $i$, and $n$ is the number of species in the

21 considered community. In other words, considering a set of species present in a community and

22 their variety of individual performances on a range of sources, the niche from that community

23 will correspond to sum of the best performances on each source present on the environment 
1 where that community is functioning. We used the maximum or best performances because this is

2 the activity level that could be achieved if the corresponding species would become dominant in

3 the community (referred to as selection or dominance effect in biodiversity studies, see Loreau

4 and Hector 2001, or Fox 2005, respectively). Community niche was used in the statistical

5 analyses, together with species richness and composition to test for their effect on community

6 functioning (see below). The performances of species on individual carbon sources used to

7 calculate community niche are listed in Appendix B.

9 Data analysis

10 General linear models (GLMs) were used to evaluate the effects of community niche,

11 bacterial richness (log scale), contrasts for the presence of particular bacterial taxa, composition

12 (different combinations of bacterial taxa) and cell density on bacterial community functions at 8

13 days after starting the microcosms. This duration corresponds to about 13 bacterial generations

14 (see second paragraph of Results section). Alternative models in which the richness term or the

15 contrasts for particular bacterial taxa were fitted before community niche were also analyzed

16 (data not shown). Selection of particular species contrasts was done by forward inclusion and

17 backward elimination using the significance level $\mathrm{P}=0.05$. Results were summarized in analysis

18 of variance (ANOVA) tables (Table 1). In these, contrasts for the presence/absence of particular

19 species were ordered in the sequence in which they were entered into the model during the

20 forward selection process. Path analyses were used to test causal relationships between

21 community niche, species richness and community processes (denitrification and $\mathrm{CO}_{2}$

22 production). The path analyses were calculated based on correlation matrices between

23 explanatory terms and the dependent variables. 
16 (mean \pm s.e.m), indicating that the bacterial communities on average had turned over $13.2 \pm 2.2$

17 times during this time period. Thus, the positive relationship observed between initial richness

18 and community processes reflected a long-term outcome after multi-generation interactions

19 between and within species. As a consequence, community composition might have changed

20 during the experiment and thus realized species richness at the end of the experiment might have

21 differed from initial species richness. Although realized richness could not be assessed in this

22 study, we did measure total bacterial abundance at the end of the experiment and found that this

23 covariate did not influence the measured community processes, denitrification and $\mathrm{CO}_{2}$

24 production (Table 1). 
2 Effects of community niche versus other components of biodiversity on functioning

3 The performances of species on individual carbon sources were used to calculate

4 community niche for denitrification $\left(\mathrm{CN}_{\mathrm{N} 2 \mathrm{O}}\right)$ and for anaerobic $\mathrm{CO}_{2}$ production $\left(\mathrm{CN}_{\mathrm{CO} 2}\right)$, for each

5 bacterial assemblage used as inoculum in the experiment. A positive linear relationship was

6 observed between community niche and the two community processes (Fig 3), indicating the

7 importance of the observed complementarity in resource use capacity among species. Fitting

8 community niche before species richness in the GLM demonstrated that $\mathrm{CN}_{\mathrm{N} 2 \mathrm{O}}$ and $\mathrm{CN}_{\mathrm{CO} 2}$ fully

9 accounted for the positive effects of species richness on community denitrification and $\mathrm{CO}_{2}$

10 production, respectively (Table 1). Moreover, the explanatory power of the community niche

11 terms was much larger than that of richness if these terms were each fitted first in separate

12 GLMs: $46 \%$ of the variance for denitrification and $32 \%$ of the variance for $\mathrm{CO}_{2}$ production was

13 explained by $\mathrm{CN}_{\mathrm{N} 2 \mathrm{O}}$ and $\mathrm{CN}_{\mathrm{CO} 2}$, respectively (Table 1). It is interesting to notice that there was

14 only a weak overlap among the functionally important species identified during the GLM

15 analyses for denitrification versus $\mathrm{CO}_{2}$ production (Table 1).

16 Graphical examples of interactions between species are provided in Figure 4. Interactions

17 between species were most often found to be positive and explained by the pattern of resource

18 utilization (Fig. 4c) whereas negative interactions were also observed (Fig. 4d) although less

19 frequently. It should be noted, however, that these results are based on single observations, as

20 assemblages with the same composition were not replicated. Our results thus provide indication

21 on the role of species interactions, but further experiments are needed to quantify this role.

22 Community niche and species contrasts together explained $68 \%$ and $69 \%$ of the variation

23 in denitrification and $\mathrm{CO}_{2}$ production, respectively. When community niche was fitted after

24 species richness, community niche still explained $30 \%$ and $13 \%$ of the variation in community 
1 denitrification and $\mathrm{CO}_{2}$ production, respectively. When $\mathrm{CN}_{\mathrm{N} 2 \mathrm{O}}$ was used as an explanatory term

2 in the GLM for community $\mathrm{CO}_{2}$ production, or $\mathrm{CN}_{\mathrm{CO} 2}$ as an explanatory term in the GLM for

3 community denitrification, their explanatory power was more than halved, demonstrating that the

4 best predictor for a particular process is indeed the measure of community niche directly

5 associated with that process.

6 The relationship among the different components of biodiversity and the measured

7 community processes was evaluated by path analysis (Fig. 5). For both processes, the effect of

8 species richness operated mainly through community niche. When entered after community niche

9 into the statistical model, richness had a weak negative and positive effect on denitrification and

$10 \quad \mathrm{CO}_{2}$ production, respectively.

\section{DisCUSSION}

13 In this study we took advantage of the tractability of the bacterial model systems to

14 evaluate the role of niche differentiation among species for biodiversity-ecosystem functioning

15 relationships (Leibold and McPeek 2006). We hypothesized that by defining an index for the

16 breadth of the niche occupied by a community, here called community niche, we should be able

17 to predict ecosystem processes which are related to resource extraction by that community in a

18 given multiple-resource environment.

19 The question of whether bacterial diversity matters to ecosystem processes has been

20 previously addressed (Wohl et al. 2004, Bell et al. 2005, Wertz et al. 2006, Jiang 2007, Wertz et

21 al. 2007), and the results range from negative or neutral to positive relationships. Jiang et al.

22 (2008) have recently argued that a positive relationship between biodiversity and ecosystem

23 functioning may not be a general trend. They suggested that negative selection (where

24 competitively dominant species do not contribute significantly to the function of interest) is more 
1 likely to operate for non-biomass ecosystem processes, leading thus to neutral or even negative

2 biodiversity-ecosystem functioning relationships (Jiang 2007, Jiang et al. 2008). Our findings do

3 not support this hypothesis, as species richness did have a positive effect on denitrification and

$4 \mathrm{CO}_{2}$ production, two ecosystem processes not entirely dependent on biomass production.

5 Moreover, denitrifying bacterial species differ greatly with respect to their specific activities (i.e.

6 activity per cell), and an indication of these differences can be seen in Fig. 2, where large

7 variations in denitrification (and $\mathrm{CO}_{2}$ production) are observed for the monocultures. Although

8 these values do not represent the specific activities of the cultures, they give an indication of it,

9 because cell density did not significantly vary among cultures (GLM analysis). A similar

10 reasoning can be applied for $\mathrm{CO}_{2}$ production, where species might have different catabolic

11 abilities. Given our findings, we suggest that additional bacterial biodiversity-ecosystem

12 functioning experiments, targeting a range of functions, are necessary to determine whether and

13 which generalizations can be made.

14 Despite its significance, species richness had a relatively low explanatory power in our

15 study, which is typical for a large number of biodiversity experiments (Balvanera et al. 2006,

16 Weigelt et al. 2008) and indicates that further components of biodiversity affect the variance in

17 bacterial community processes. This was confirmed by including community niche in the

18 analysis, which greatly expanded the explained variance. An additional part of the variation in

19 community processes could be explained by the presence or absence of particular species,

20 indicating that effects other than the capacity to use the carbon sources also played a role in our

21 experiment, as exemplified in Fig. 4d. However, identifying the mechanisms generating the

22 effects of particular bacterial species (production of antibiotics, quorum sensing signals, etc.) was

23 beyond the scope of this study. Furthermore, it is conceivable that species behaved differently in

24 mixtures than in monoculture and this might be an additional reason for unexplained variance in 
1 our experiment. Finally, as time went by in this multi-generation experiment, other resources may

2 have become available through the release of bacterial metabolites or due to cell death.

3 Nevertheless, path analysis demonstrated that community niche was the main driver for the

4 corresponding community process and provided evidence that species richness influenced

5 denitrification and $\mathrm{CO}_{2}$ production only indirectly via its influence on community niche.

6 The positive linear relationships between community niche and functioning for both

7 processes studied indicated that the larger the community niche of a given community, the more

8 efficient it can exploit the available resources and the better it performs in the corresponding

9 process. This finding led us to the conclusion that the pattern of resource utilization by individual

10 species and among species was the major effect explaining enhanced collective performance of

11 diverse communities. Attempts to provide a mechanistic interpretation of bacterial diversity-

12 ecosystem functioning experiments suggest that complementarity (through resource partitioning

13 and/or facilitation) plays a more important role than selection effect, when functions related to

14 biomass production were evaluated (Wohl et al. 2004, Bell et al. 2005, Jiang 2007). An open

15 question is whether it is relevant to disentangle the role of selection and complementarity in

16 multi-generation biodiversity experiments. We argue that for microbial communities, widely-

17 used mechanistic interpretation of biodiversity-ecosystem functioning such as those based on

18 additive partitioning methods (Loreau and Hector 2001, Fox 2005) seem less appropriate since

19 population dynamics leads to potentially strong shifts in individual species abundances along a

20 time scale. Moreover, in these experiments, niche partitioning leads to transient dynamics which

21 reflects evolutionary responses resulting from both selection and complementarity effects. We

22 propose that in the case of dynamic communities, indices based on the effects of species on

23 ecosystem processes are more appropriate as they go beyond these interpretations, and yet

24 provide a mechanistic explanation. This is the case for community niche, but also indexes based 
1 on the functional dissimilarity between species (Heemsbergen et al. 2004). All these indices

2 could be used, due to their deductive reasoning, to predict how communities influence ecosystem

3 processes prior to or without experimentation.

$4 \quad$ Our conclusions cannot be directly generalized to natural environments often characterized

5 by very large bacterial diversity that can lead to high functional redundancy (Wertz et al. 2006,

6 2007). Rather, our study provides a proof of concept how measures of community resource use

7 may be used to predict ecosystem functioning under ideal conditions. It remains to be tested how

8 well such predictions will match with the more complex situation in the field. Although we used

9 bacteria to test our hypothesis, our approach could be extended to other organisms such as plants,

10 for which the best choice of functional diversity measures remains a challenge (Lavorel and

11 Garnier 2002, Naeem and Wright 2003, Petchey and Gaston 2006). For instance, the

12 characterization of the uptake rates of different nitrogen compounds in soil such as nitrate,

13 ammonium and small organic nitrogen compounds by a range of plant species could be used to

14 determine a community niche for different plant communities and predict plant diversity effects

15 on ecosystem processes such as overall $\mathrm{N}$ uptake from soil or primary production. Furthermore,

16 for experiments reflecting short-term dynamics, the already high explanatory power of this index

17 might be further enhanced if the performance of each species could be weighed by its actual

18 abundance in each community.

19

20 ACKNOWLEDGMENTS

21 We thank T. Bell, M. Loreau, N. Mouquet and two anonymous reviewers for their valuable

22 comments on earlier versions of the manuscript, G. Mouchiroud for use of the flow cytometer,

23 and the graduate students who helped in the characterization of species niches. J.F.S. was 
1 supported by a post doctoral grant from the Institut National de la Recherche Agronomique

2 (INRA). B.S. was supported by a grant from the Swiss Science Foundation (grant 31-65224.01).

4 LiTERATURE CITED

5 Balvanera, P., A. B. Pfisterer, N. Buchmann, J.-S. He, T. Nakashizuka, D. Raffaelli, and B.

6 Schmid. 2006. Quantifying the evidence for biodiversity effects on ecosystem functioning and $7 \quad$ services. Ecology Letters 9:1146-1156.

8 Bathe, S., W. Achouak, A. Hartmann, T. Heulin, M. Schloter, and M. Lebuhn. 2006. Genetic and 9 phenotypic microdiversity of Ochrobactrum spp. FEMS Microbiology Ecology 56:272-280.

10 Bell, T., J. A. Newman, B. W. Silverman, S. L. Turner, and A. K. Lilley. 2005. The contribution 11 of species richness and composition to bacterial services. Nature 436:1157-1160.

12 Cavigelli, M. A., and G. P. Robertson. 2001. Role of denitrifier diversity in rates of nitrous oxide 13 consumption in a terrestrial ecosystem. Soil Biology and Biochemistry 33:297-310.

14 Fox, J. W. 2005. Interpreting the 'selection effect' of biodiversity on ecosystem function. Ecology 15 Letters 8:846-856.

16 Heemsbergen, D. A., M. P. Berg, H. A. Verhoef, J. R. Van Hal, J. H. Faber, and M. Loreau.

17 2004. Biodiversity effects on soil processes explained by interspecific functional dissimilarity. 18 Science 306:1019-1020.

19 Jessup, C. M., R. Kassen, S. E. Forde, B. Kerr, A. Buckling, P. B. Rainey, and B. J. M.

20 Bohannan. 2004. Big questions, small worlds: microbial model systems in ecology. Trends in $21 \quad$ Ecology \& Evolution 19:189-197.

22 Jiang, L. 2007. Negative selection effects suppress relationships between bacterial diversity and 23 ecosystem fucntioning. Ecology 88:1075-1085. 
1 Jiang, L., Z. Pu, and N. D. R. 2008. On the importance of the negative selection effect for the

2 relationship between biodiversity and ecosystem functioning. Oikos 117:488-493.

3 Lavorel, S., and E. Garnier. 2002. Predicting changes in community composition and ecosystem

4 functioning from plant traits: revisiting the Holy Grail. Functional Ecology 16:545-556.

5 Leibold, M. A. 1995. The Niche Concept Revisited - Mechanistic Models and Community

$6 \quad$ Context. Ecology 76:1371-1382.

7 Leibold, M. A., and M. A. McPeek. 2006. Coexistence of the niche and neutral perspectives in

$8 \quad$ community ecology. Ecology 87:1399-1410.

9 Loreau, M., and A. Hector. 2001. Partitioning selection and complementarity in biodiversity

10 experiments. Nature 412:72-76.

11 Naeem, S., D. R. Hahn, and G. Schuurman. 2000. Producer-decomposer co-dependency

12 influences biodiversity effects. Nature 403:762-764.

13 Naeem, S., and J. P. Wright. 2003. Disentangling biodiversity effects on ecosystem functioning:

14 deriving solutions to a seemingly insurmountable problem. Ecology Letters 6:567-579.

15 Petchey, O. L., and K. J. Gaston. 2006. Functional diversity: back to basics and looking forward.

16 Ecology Letters 9:741-758.

17 Philippot, L., and S. Hallin. 2005. Finding the missing link between diversity and activity using

18 denitrifying bacteria as a model functional community. Current Opinion in Microbiology

19 8:234-239.

20 Prosser, J. I., B. J. M. Bohannan, T. P. Curtis, R. J. Ellis, M. K. Firestone, R. P. Freckleton, J. L.

21 Green, L. E. Green, K. Killham, J. J. Lennon, A. M. Osborn, M. Solan, C. J. van der Gast, and

22 J. P. W. Young. 2007. The role of ecological theory in microbial ecology. Nature Reviews

23 Microbiology 5:384-392. 
1 Sambrook, J., E. F. Fritisch, and T. Maniatis. 1989. Molecular cloning: a labaratory manual,

2 second edition. Cold Spring Harbor labaratory press, Cold Spring Harbor.

3 Tilman, D. 1982. Resource competition and community structure. Princeton University Press,

4 Princeton, NJ.

5 Weigelt, A., J. Schumacher, C. Roscher, and B. Schmid. 2008. Does biodiversity increase spatial

$6 \quad$ stability in plant community biomass? Ecology Letters 11:338-347.

7 Wertz, S., V. Degrange, J. I. Prosser, F. Poly, C. Commeaux, T. Freitag, N. Guillaumaud, and X.

8 Le Roux. 2006. Maintenance of soil functioning following erosion of microbial diversity.

$9 \quad$ Environmental Microbiology 8:2162-2169.

10 Wertz, S., V. Degrange, J. I. Prosser, F. Poly, C. Commeaux, N. Guillaumaud, and X. Le Roux.

11 2007. Decline of soil microbial diversity does not influence the resistance and resilience of key

12 soil microbial functional groups following a model disturbance. Environmental Microbiology

$13 \quad 9: 2211-2219$.

14 Wohl, D. L., S. Arora, and J. R. Gladstone. 2004. Functional redundancy supports biodiversity

15 and ecosystem function in a closed and constant environment. Ecology 85:1534-1540.

16 
1 Table 1. Results of GLM analyses presenting the effects of community niche, species richness,

2 contrasts for the presence of particular species, community composition (different combinations

3 of species) and cell density on bacterial community functions 8 days after inoculating the

4 microcosms. Significant terms are in bold.

\begin{tabular}{|c|c|c|c|c|c|c|c|c|c|}
\hline Denitrification & d.f. & $F$ & $P$ & $\%$ s.s. & $\mathrm{CO}_{2}$ production & d.f. & $F$ & $P$ & $\%$ s.s. \\
\hline $\begin{array}{l}\text { Community } \\
\text { niche }\end{array}$ & 1 & 99.72 & 0.0000 & 46.22 & Community niche & 1 & 66.47 & 0.0000 & 31.90 \\
\hline Species richness & 1 & 0.44 & $\mathrm{~ns}$ & 0.20 & Species richness & 1 & 1.44 & ns & 0.69 \\
\hline $\begin{array}{l}\text { Citrobacter } \\
\text { braakii A7 }\end{array}$ & 1 & 14.58 & 0.0004 & 6.76 & $\begin{array}{l}\text { Pseudomonas } \\
\text { aeruginosa G16 }\end{array}$ & 1 & 10.47 & 0.0021 & 5.03 \\
\hline $\begin{array}{l}\text { Ochrobactrum } \\
\text { sp. A17 }\end{array}$ & 1 & 9.28 & 0.0036 & 4.30 & $\begin{array}{l}\text { Ochrobactrum sp. } \\
\text { A6 }\end{array}$ & 1 & 16.61 & 0.0002 & 7.97 \\
\hline $\begin{array}{l}\text { Pseudomonas } \\
\text { stutzeri A24 }\end{array}$ & 1 & 9.09 & 0.0040 & 4.21 & $\begin{array}{l}\text { Ensifer adhaerens } \\
\text { A1 }\end{array}$ & 1 & 13.27 & 0.0006 & 6.37 \\
\hline $\begin{array}{l}\text { Ochrobactrum } \\
\text { sp. A22 }\end{array}$ & 1 & 6.03 & 0.017 & 2.80 & $\begin{array}{l}\text { Ochrobactrum sp. } \\
\text { A22 }\end{array}$ & 1 & 16.17 & 0.0002 & 7.76 \\
\hline $\begin{array}{l}\text { Pseudomonas } \\
\text { aeruginosa G16 }\end{array}$ & 1 & 3.06 & 0.0847 & 1.43 & $\begin{array}{l}\text { Bacillus } \\
\text { weihenstephanensis } \\
\text { A20 }\end{array}$ & 1 & 4.25 & 0.044 & 2.04 \\
\hline $\begin{array}{l}\text { Klebsiela } \\
\text { pneumoniae A18 }\end{array}$ & 1 & 4.49 & 0.039 & 2.08 & $\begin{array}{l}\text { Pseudomonas } \\
\text { stutzeri A24 } \\
\text { Azospirillum } \\
\text { lipoferum A5 }\end{array}$ & 1 & 8.27 & 0.0063 & 3.97 \\
\hline Total species & 6 & 7.76 & 0.0000 & 21.58 & Total species & 7 & 11.02 & 0.0000 & 37.02 \\
\hline Composition & 52 & 1.10 & ns & 24.10 & Composition & 51 & 1.63 & $\mathrm{~ns}$ & 24.48 \\
\hline
\end{tabular}


$\begin{array}{llllllllll}\text { Cell density } & 1 & 1.81 & \text { ns } & 0.76 & \text { Cell density } & 1 & 3.21 & \text { ns } & 0.95\end{array}$

1 Note that species contrasts cannot be ordered in sequence of decreasing explanatory power because some species

2 explain more variation if they are fitted after than before other species. Therefore, we used the sequence in which

3 species were entered into the model during the forward selection process. d.f. $=$ degrees of freedom. $\%$ s.s. $=$

4 percentage of the total sum of squares explained by each variable. ns $=$ not significant $(P>0.05)$.

5

6 


\section{Figure LEGENDS}

2 Figure 1: PCA analysis based on the relative performance $\left(\mathrm{N}_{2} \mathrm{O}\right.$ production) of each species on

3 each carbon source. Diamonds represent the bacterial species; arrows are projected vectors

4 corresponding to carbon sources: C1, D-cellobiose; C2, maltose; C3, L-fucose; C4, L-malic acid;

5 C5, L-glutamine and C6, fumaric acid. The first and the second axes accounted for 52.4 and

$6 \quad 17.8 \%$ of total variance, respectively.

8 Figure 2: Relationship between the functioning and species richness of bacterial communities.

9 Two processes were studied: (a) denitrification and (b) $\mathrm{CO}_{2}$ production under anaerobiosis. Each

10 diamond represents a single community, and asterisks represent mean values at each richness

11 level. Regression lines are linear fits with log (species richness) as independent variable,

12 excluding the highest richness level (16 species of the initial pool). Note that two points are

13 confounded for the 16 species level.

15 Figure 3: Relationship between community functioning and community niche. Community niche

16 was rescaled by dividing by the maximum value observed for all communities: (a) denitrification

17 as a function of community niche defined according to monoculture ability to perform

18 denitrification on each individual carbon source; (b) anaerobic $\mathrm{CO}_{2}$ production as a function of

19 community niche defined according to monoculture ability to produce $\mathrm{CO}_{2}$ on each individual

20 carbon source. Different colors correspond to different levels of species richness. Each symbol

21 corresponds to a single community, except for the monocultures, which are represented by their

22 average performances. The graph shows that communities with low richness but large community

23 niche perform better than communities with high richness but small community niche. 
$1 \quad$ Figure 4: Example of complementarity and of negative interaction between species.

2 Complementarity was observed between two species (G11: Paracoccus denitrificans and G16:

3 Pseudomonas aeruginosa) that differ in their ability to produce $\mathrm{N}_{2} \mathrm{O}$ on different carbon sources

4 (a); in that case, the performance of the mixture of the 2 species (grey diamond) is higher than the

5 performance of each monoculture (black diamonds) which is adequately predicted by the change

6 in community niche (c). Negative interaction was observed between two other species (A20:

7 Bacillus weihenstephanensis and A24: Pseudomonas stutzeri). In that case, independently of the

8 differences in their ability to produce $\mathrm{N}_{2} \mathrm{O}$ on different carbon sources (b), the performance of the

9 mixture of the 2 species is lower than the performance of each monoculture (d) which indicates

10 negative interactions such as antibiotics or inhibitory compounds production.

12 Figure 5: Relative effect of bacterial richness and community niche on community functioning.

13 Path analyses were used to evaluate the roles of these different aspects of biodiversity for (a)

14 denitrification and (b) anaerobic $\mathrm{CO}_{2}$ production. The values not linked to any variable indicate

15 that amount of variance that remains unexplained.

16 


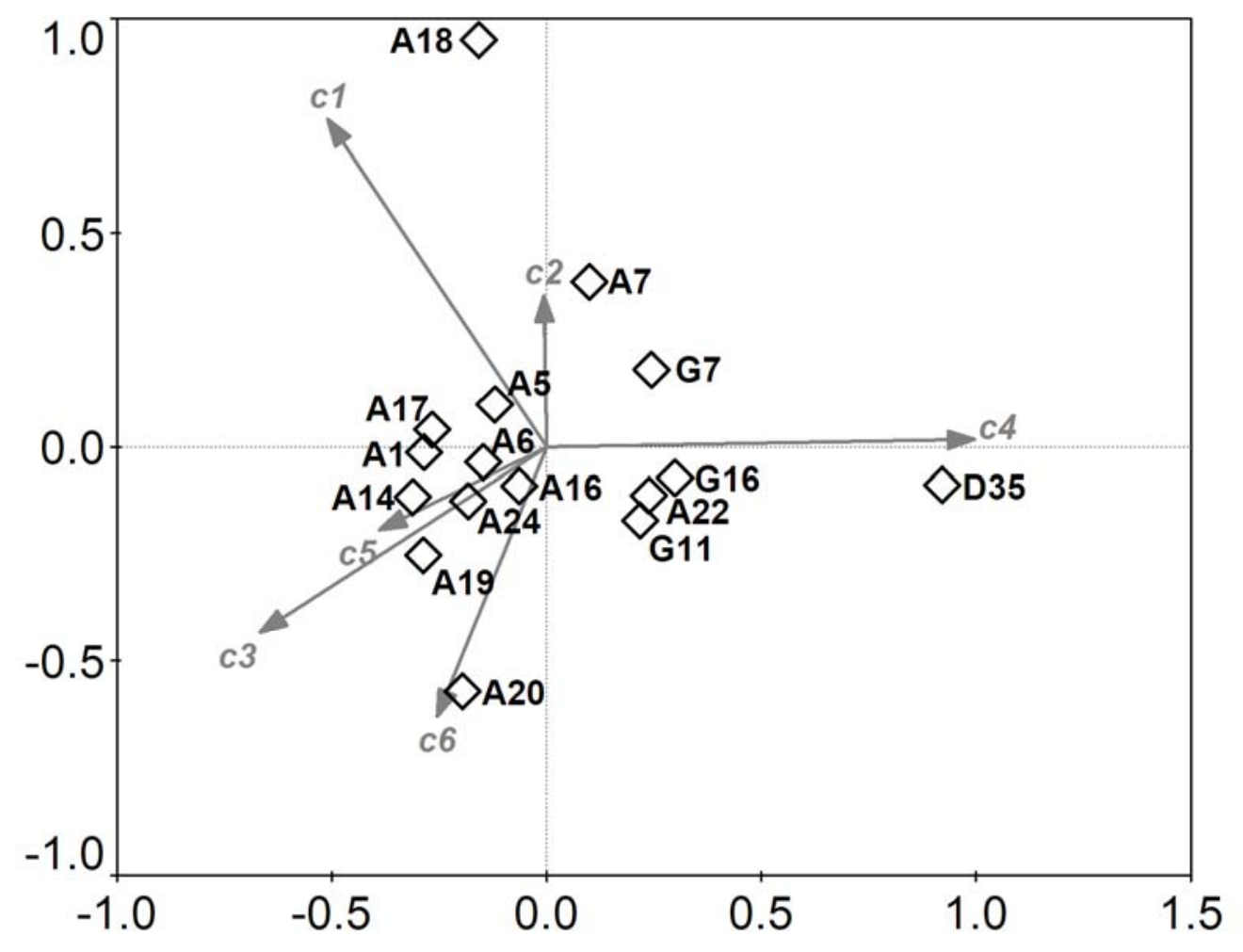

$4 \quad$ Figure 1

5

6

7

8

9

10

11

12 


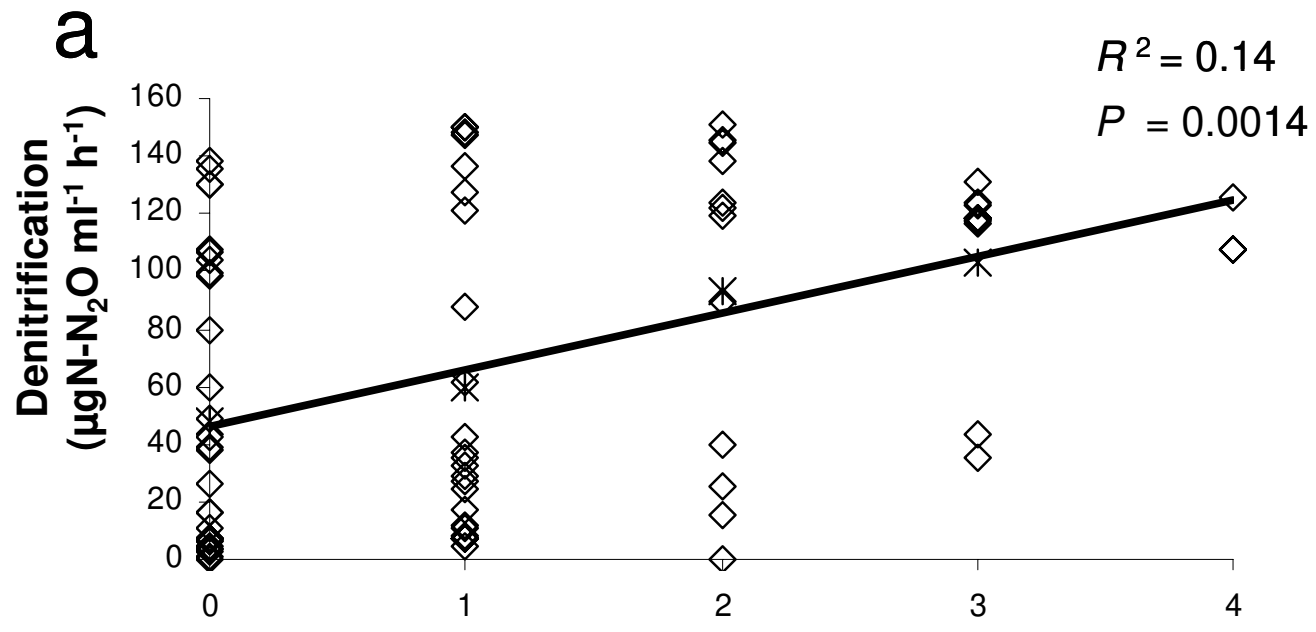

10

b

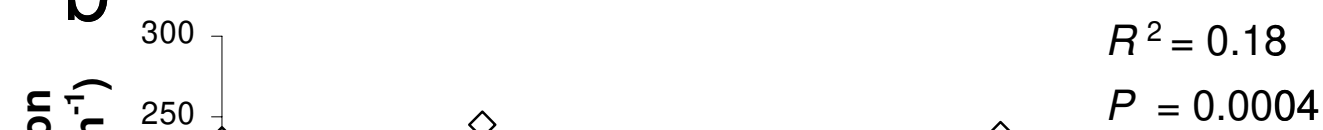

12

13

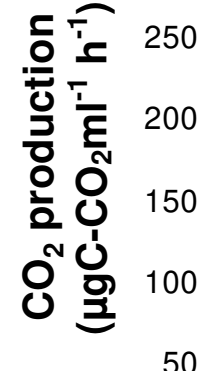

$18 \quad$ Figure 2

19 
a
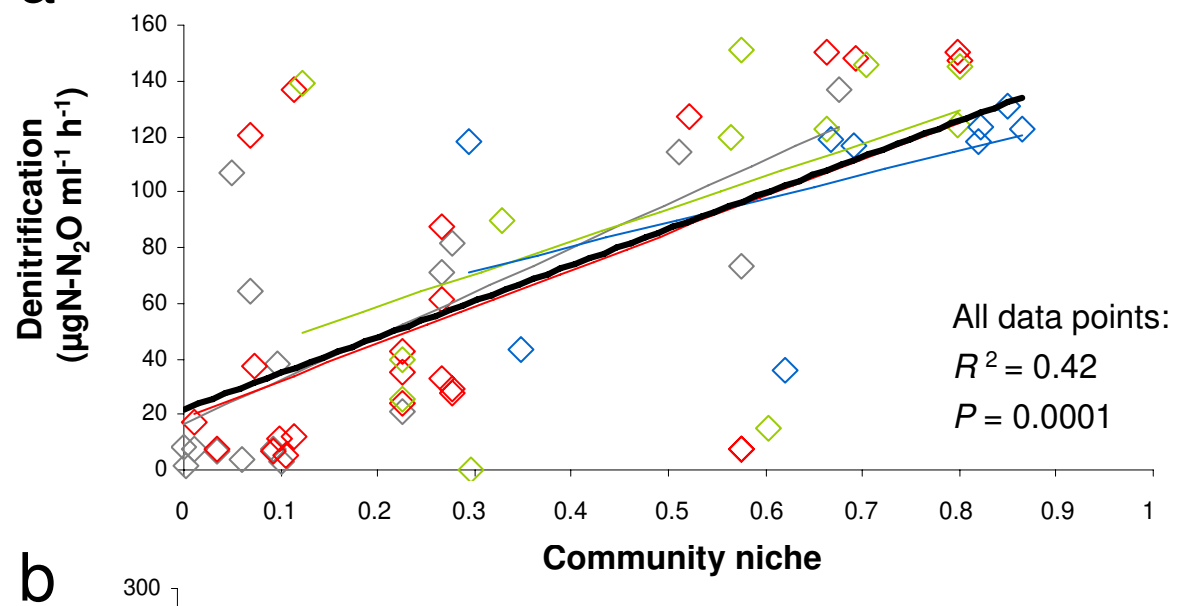

\section{$15 \quad$ Figure 3}

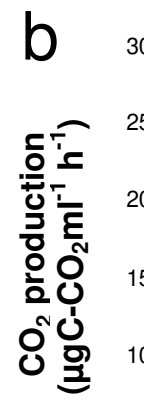

50
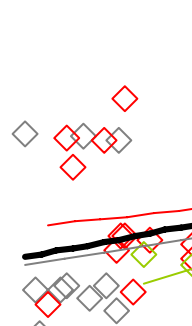
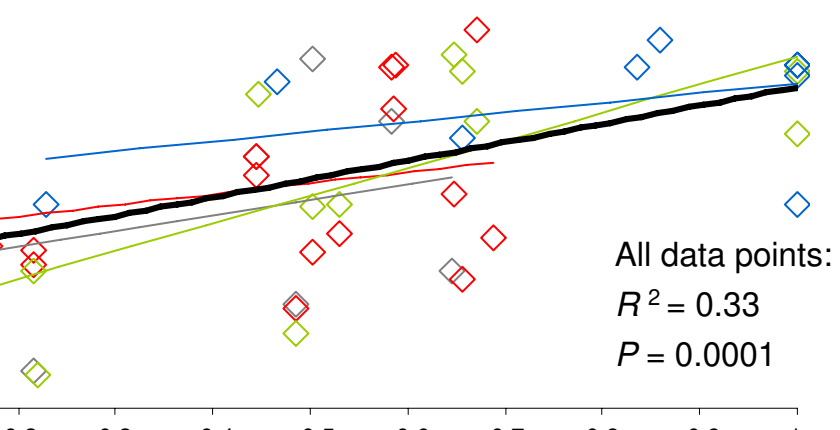

All data points:

$R^{2}=0.33$

$P=0.0001$

16

17

18

19

20

21 
2

3

4

5

6

7

8

9

10

11

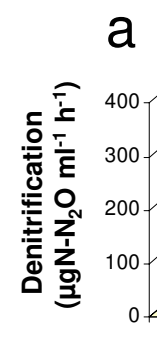

a

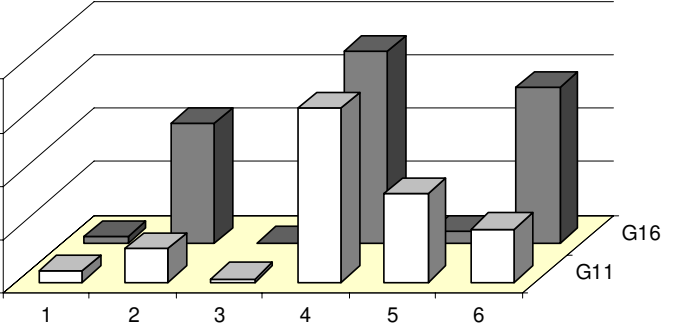

Individual carbon sources
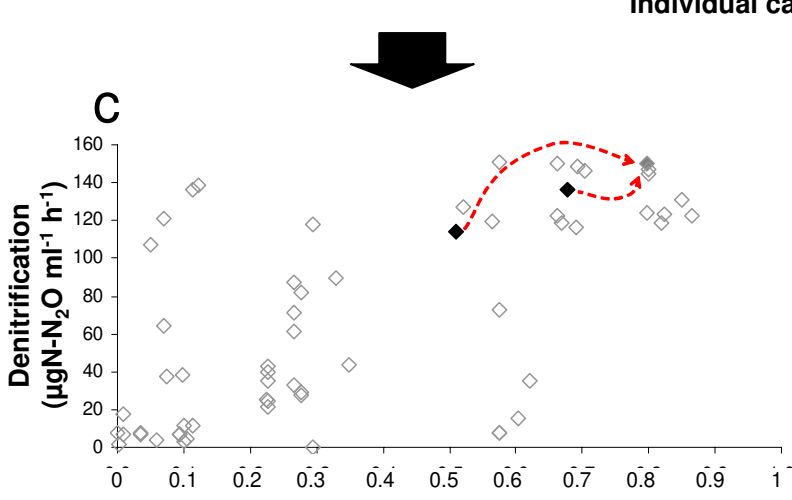
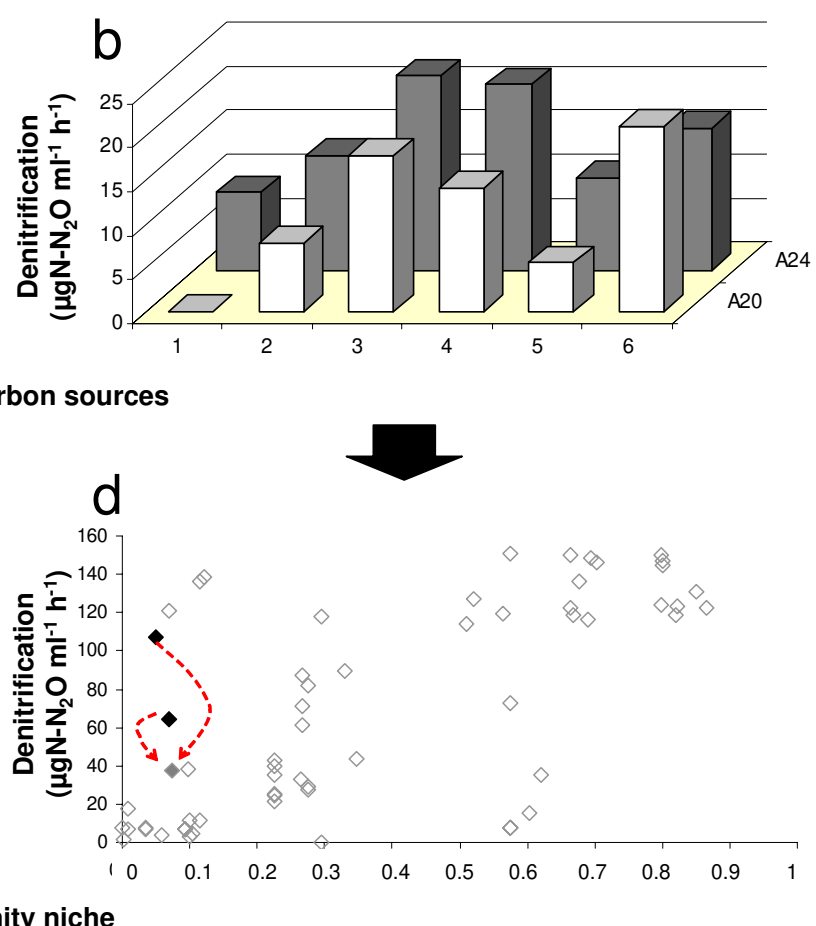

12

\section{$13 \quad$ Figure 4}


1

2

3

4

5

6

7

8

9

10

11

12

13

14

$15 \quad$ Figure 5 a

Species richness

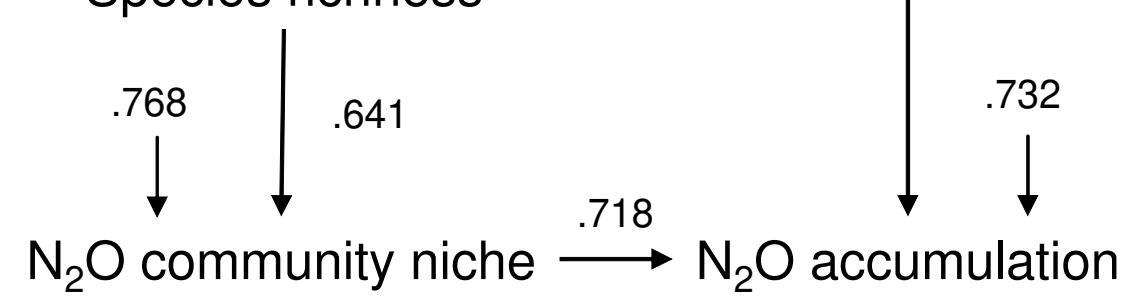

b

Species richness

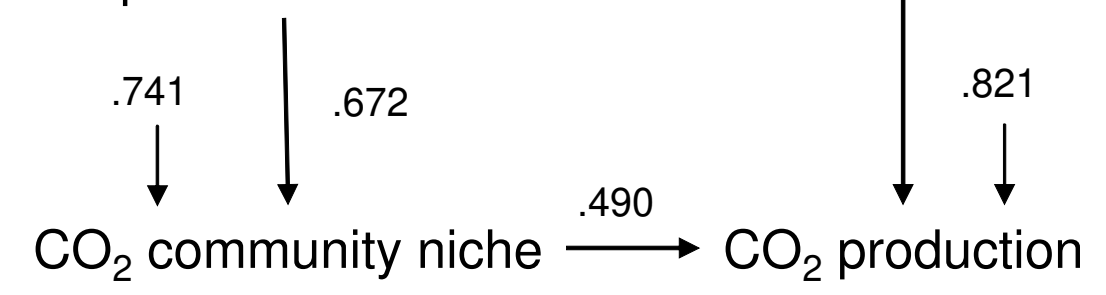

.112 